\title{
Lysine methyltransferase SMYD2 promotes triple negative breast cancer progression
}

\author{
Linda Xiaoyan Li ${ }^{1}$, Julie Xia Zhou', James P. Calvet ${ }^{2,3}$, Andrew K. Godwin ${ }^{4}$, Roy A. Jensen (1) and Xiaogang Li ${ }^{1,2,3}$
}

\begin{abstract}
We identified SMYD2, a SMYD (SET and MYND domain) family protein with lysine methyltransferase activity, as a novel breast cancer oncogene. SMYD2 was expressed at significantly higher levels in breast cancer cell lines and in breast tumor tissues. Silencing of SMYD2 by RNAi in triple-negative breast cancer (TNBC) cell lines or inhibition of SMYD2 with its specific inhibitor, AZ505, significantly reduced tumor growth in vivo. SMYD2 executes this activity via methylation and activation of its novel non-histone substrates, including STAT3 and the p65 subunit of NF-KB, leading to increased TNBC cell proliferation and survival. There are cross-talk and synergistic effects among SMYD2, STAT3, and NF-KB in TNBC cells, in that STAT3 can contribute to the modification of NF-KB p65 subunit post-translationally by recruitment of SMYD2, whereas the p65 subunit of NF-KB can also contribute to the modification of STAT3 post-translationally by recruitment of SMYD2, leading to methylation and activation of STAT3 and p65 in these cells. The expression of SMYD2 can be upregulated by IL-6-STAT3 and TNFa-NF-KB signaling, which integrates epigenetic regulation to inflammation in TNBC development. In addition, we have identified a novel SMYD2 transcriptional target gene, PTPN13, which links SMYD2 to other known breast cancer associated signaling pathways, including ERK, mTOR, and Akt signaling via PTPN13 mediated phosphorylation.
\end{abstract}

\section{Introduction}

Triple-negative breast cancer (TNBC), in which the expression of estrogen receptor (ER), progesterone receptor and human epidermal growth factor receptor 2 are lacking, is a common and aggressive subtype of breast cancer with poor prognostic outcome and reduced shortterm survival compared with other types of breast cancer $^{1}$. Due to the loss of three important receptors, TNBC is more difficult to treat and more likely to recur. The challenges of TNBC are in fact more fundamental than insensitivity to current available therapeutics. A major barrier to developing TNBC therapies is our lack of understanding of the molecular drivers of TNBC. As a result, the roles of epigenetic modulation of gene

\footnotetext{
Correspondence: Xiaogang Li (xli3@kumc.edu)

${ }^{1}$ Department of Internal Medicine, University of Kansas Medical Center, Kansas City, KS 66160, USA

${ }^{2}$ Department of Biochemistry and Molecular Biology, University of Kansas Medical Center, Kansas City, KS 66160, USA

Full list of author information is available at the end of the article Edited by A. Peschiaroli
}

expression and protein function in breast cancer have become a major focus of scientific investigation ${ }^{2-4}$. Identifying the epigenetic signaling networks whose dysregulation drives TNBC would have an enormous impact on our understanding of the disease and how we treat patients.

In eukaryotic cells, genomic DNA is densely packed with histones to form chromatin. Active transcription requires local unwinding of the chromatin structure with post-translational modifications of histones to facilitate accessibility of transcription factors. Histone lysine methylation can occur at particular lysines of histone $\mathrm{H} 3$ and $\mathrm{H} 4$ to either activate or repress transcription. The accumulated evidence suggests that many histone/lysine methyltransferases function as oncogenes or tumorsuppressors to regulate cancer initiation and progres$\operatorname{sion}^{5-7}$. A SET and MYND domain-containing histone (lysine) methyltransferase, SMYD2, methylates histone $\mathrm{H} 3 \mathrm{~K} 4$ and H3K36 and non-histone breast cancer associated proteins, including $\mathrm{p} 53, \mathrm{Rb}, \mathrm{HSP} 90$ and estrogen 
receptor $\alpha(E R \alpha)^{8-13}$. SMYD2 methylates p53 to prevent p53 from binding to its target gene promoters, and knockdown of SMYD2 enhances DNA damage-induced, p53-dependent apoptosis ${ }^{10}$. SMYD2 methylates $\mathrm{Rb}$ on lysines, which results in either the repression of specific $\mathrm{Rb} / \mathrm{E} 2 \mathrm{~F}$ genes or an increase in $\mathrm{Rb}$ phosphorylation, leading to cell cycle progression ${ }^{11}$. Under estrogendepleted conditions, SMYD2 methylates ER $\alpha$ to prevent its recruitment to its target gene promoters ${ }^{13}$. SMYD2 has been reported to be overexpressed in esophageal squamous cell carcinoma (ESCC) primary tumor samples and in pediatric acute lymphoblastic leukemia correlated with a poor prognosis and patient survival ${ }^{14,15}$. Genetic knockdown of SMYD2 leads to decreased ESCC cell proliferation via cell cycle regulation and apoptosis ${ }^{14}$. Quantitative reverse transcription PCR (qRT-PCR) analysis indicated that SMYD2 mRNA levels in 14 out of 20 breast cancer cell lines were increased at least two-fold compared to those in MCF10A cells, an immortalized but non-tumorigenic breast epithelial cell line ${ }^{4}$. However, the roles and mechanisms by which SMYD2 promotes cancer progression remain unknown.

In this study, we found that SMYD2 promotes triplenegative breast cancer development via the synergistic methylation and activation of its specific non-histone substrates, STAT3 and NF- $\mathrm{kB}$, and via the methylation of histones to transcriptionally regulate the expression of gene(s) related to cancer development. We also found that knockdown of SMYD2 and inhibition of SMYD2 with its specific inhibitor, AZ505, prevented tumor growth in TNBC cells implanted nude mice. Understanding the roles and mechanisms of SMYD2 in TNBC should make SMYD2 an attractive drug target for TNBC treatment, which lacks specific targeted therapy options at present.

\section{Results}

\section{SMYD2 is highly expressed in triple negative breast} cancers

The genetic alterations of histone methyltransferases, including SMYD2, in breast cancer were systematically investigated in breast cancer samples from the cancer genome atlas (TCGA) database via cBio Portal ${ }^{16,17}$. We found that SMYD2 was upregulated in almost all cancer types based on the cross-cancer alteration summary for SMYD2, which included 91 studies, and this was particularly true in breast cancer samples (Fig. 1a). And the protein levels of SMYD2 were increased in two TNBC cell lines, MDA-MB231, MDA-MB468 cells as well as in MCF-7 cells and T47D cells, compared to MCF10A cells in both cytoplasmic fraction and nuclear fraction (Fig. 1b). We also found that SMYD2 was localized to both the cytoplasm and nucleus in TNBC cells. (Fig. 1c).In addition, significantly elevated SMYD2 copy number was found in all subtypes of breast cancer and increased
SMYD2 levels were correlated with poor patient survival (Figs. 1d and e). In the basal-like breast cancer subtype, compared with non-basal subtypes, the expression levels of SMYD2 were significantly higher $(p<0.001)$. For SMYD3, which shares a high degree of sequence homology with SMYD2, the expression levels were significantly lower $(p<0.001)^{4}$.

\section{Knockdown of SMYD2 decreased tumor growth in mice bearing xenografts of TNBC cells}

To investigate whether SMYD2 is required for in vivo tumor growth, SMYD2 shRNA stable knockdown TNBC cells were implanted in nude mice and tumor growth was measured and compared with that in mice implanted with control vector-infected TNBC parental cells. Robust tumors developed in mice implanted with the control TNBC cells within 30 days. In contrast, tumor growth was inhibited in two SMYD2 knockdown MDA-MB468 cells (Figs. 2a, b) and MDA MB231 cells (Figs. 2c-e) as seen by a decrease in tumor sizes compared to the control mice. We also confirmed the knockdown of SMYD2 in tissues by qRT-PCR (Fig. 2f). We also found that depletion of SMYD2 decreased tumor cell proliferation assayed by Ki67 staining (Fig. 2g) and induced apoptosis by TUNEL assay (Fig. 2h). In addition, we found that overexpressing GFP-tagged SMYD2 increased cell proliferation of HEK293T cells and MCF10A cells (Supplementary Fig. S1A and 1B). These results suggested that SMYD2 functions as a regulator of tumor progression in TNBC.

\section{Inhibition of SMYD2 with AZ505 suppressed tumor growth} in mice bearing xenografts of TNBC cells

AZ505 was discovered through a high throughput chemical screen as a selective inhibitor of SMYD2 when it was found to bind the substrates binding groove of SMYD $2^{18}$. We found that AZ505 treatment induced apoptosis compared to DMSO treated cells as measured by TUNEL and FACS analysis (Figs. 3a, b). We also found that AZ505 treatment inhibited MDA-MB231 cell migration determined in a wound closure assay (Fig. $3 \mathrm{c}$ ), and treatment with AZ505 just slightly further inhibited the cell migration in SMYD2-knockdown MDAMB231cells (Fig. 3d). In addition, we found AZ505 treatment decreased S-phase entry in both MDA-MB231 and MDA-MB468 cells as examined by FACS analysis (Fig. 3e). The effect of AZ505 on TNBC cell proliferation is in a dose dependent manner as examined by MTT assay (Fig. 3f). Similar inhibition effects of AZ505 on cell proliferation and cell migration were also found in ER positive MCF-7 cells and T47D cells (Supplementary Fig. S2). Knockdown of SMYD2 also inhibited cell cycle in MDAMB231 cells, while treatment with AZ505 further inhibited cell cycle in SMYD2-knockdown cells (Fig. 3g). Treatment with AZ505 further inhibited cell proliferation 


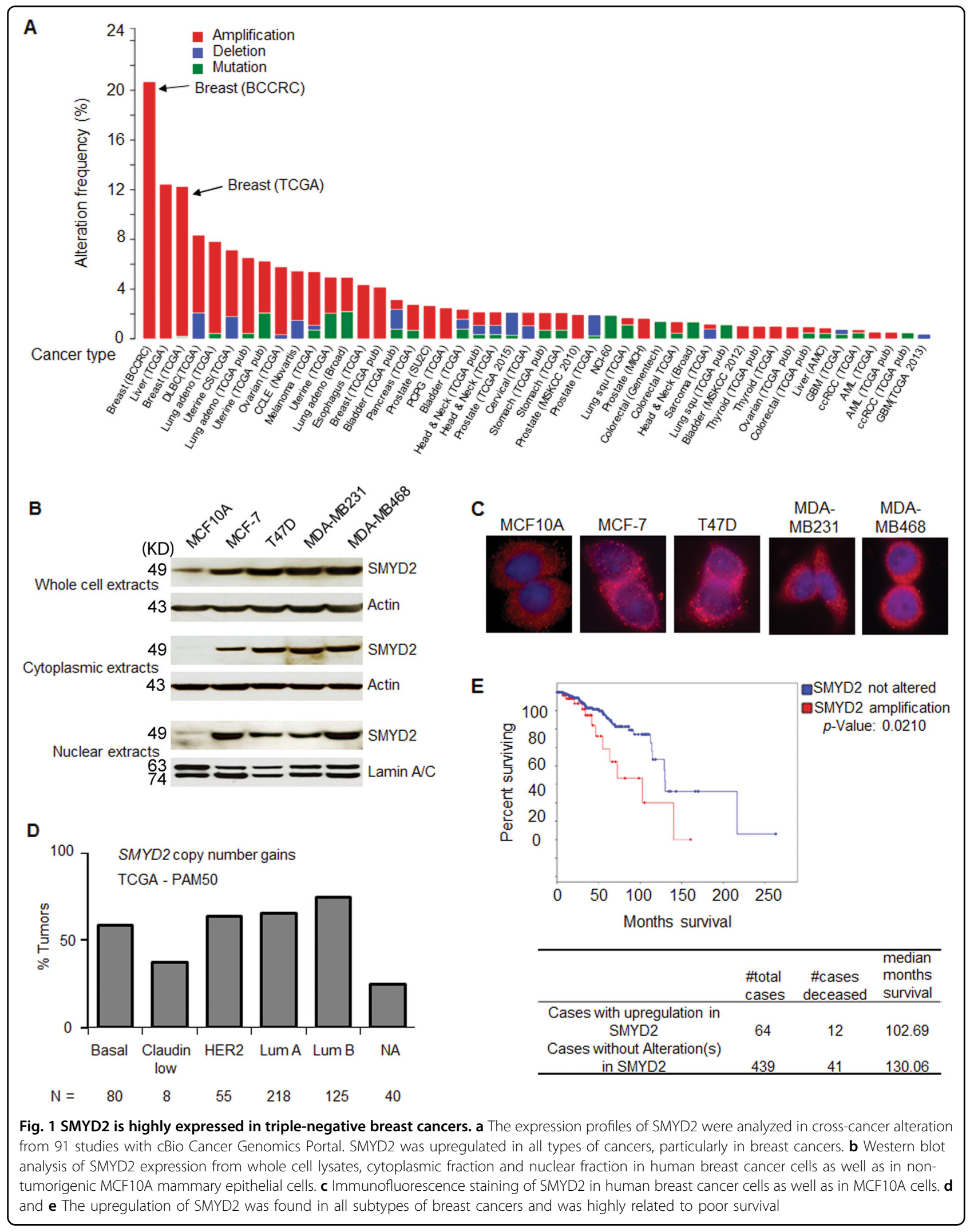




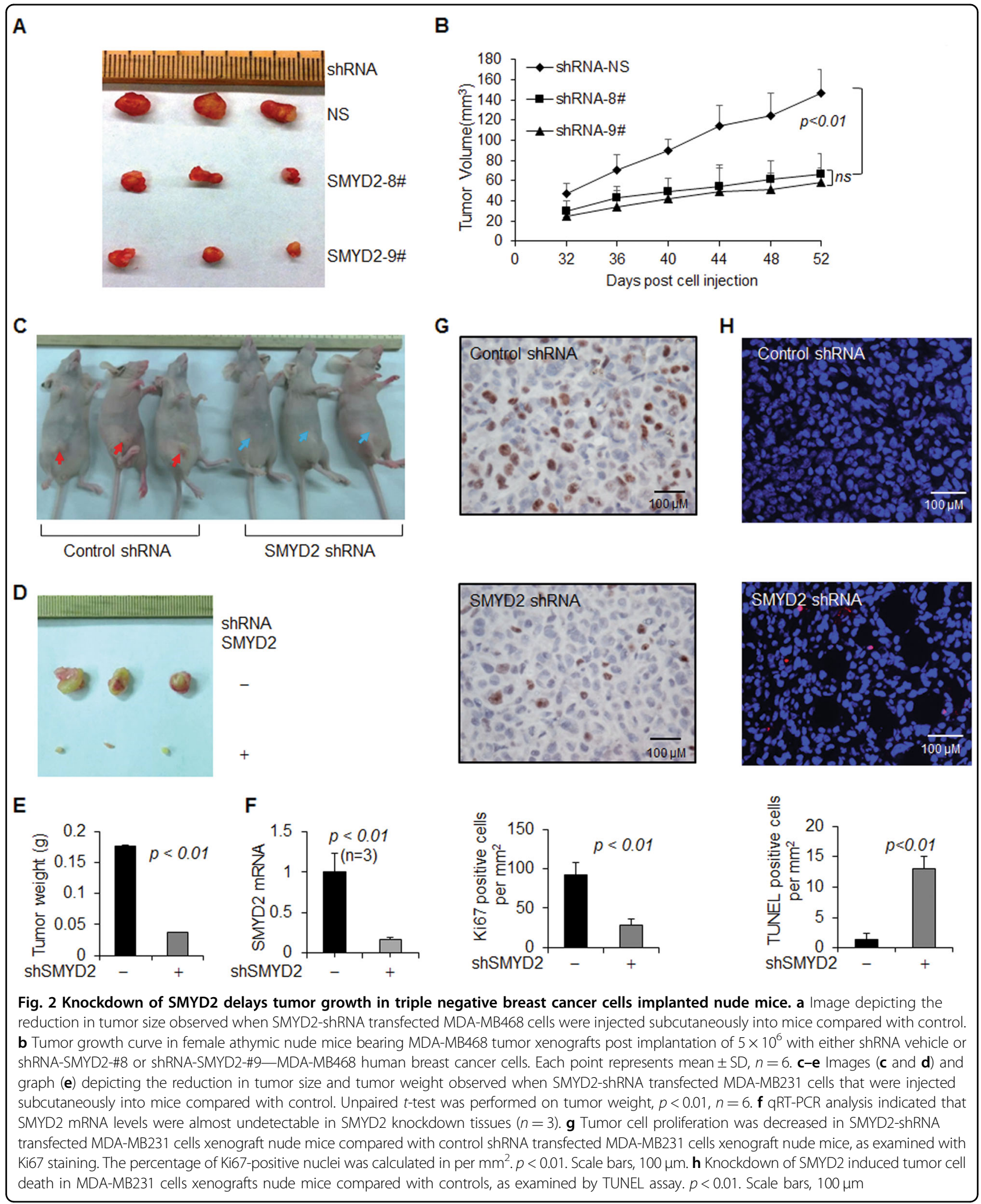

in SMYD2 knockdown MDA-MB231 cells compared to that in the DMSO treated SMYD2 knockdown cells (Fig. 3h). The results shown in Figs. 3d, g, and h might be caused by the inhibition of AZ505 on the remaining SMYD2 in SMYD2 knockdown cells since we found that treatment with AZ505 didn't further affect cell 


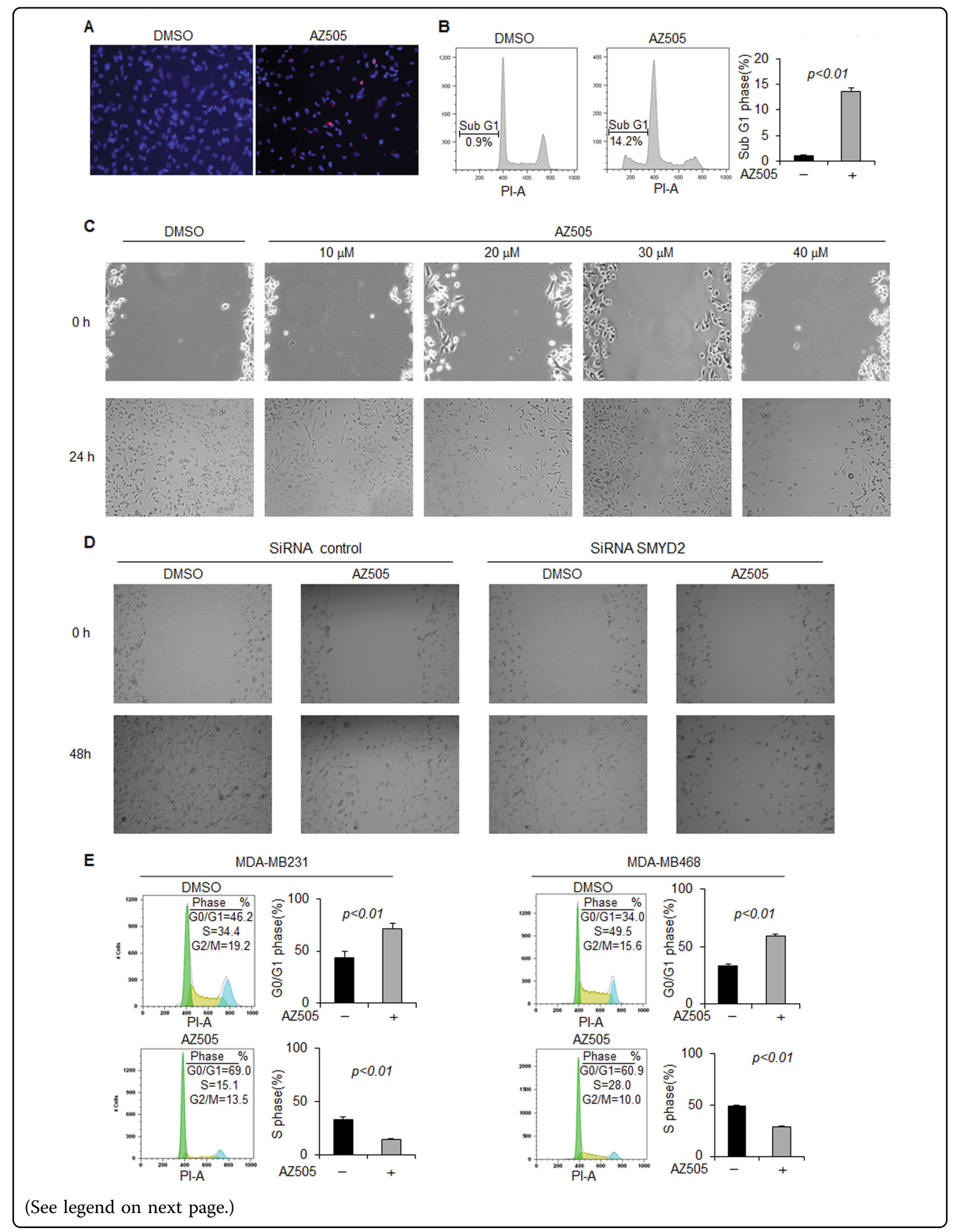



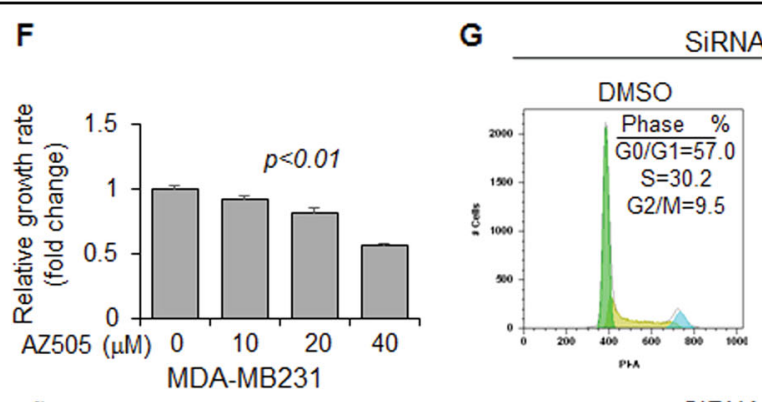

SiRNA Control
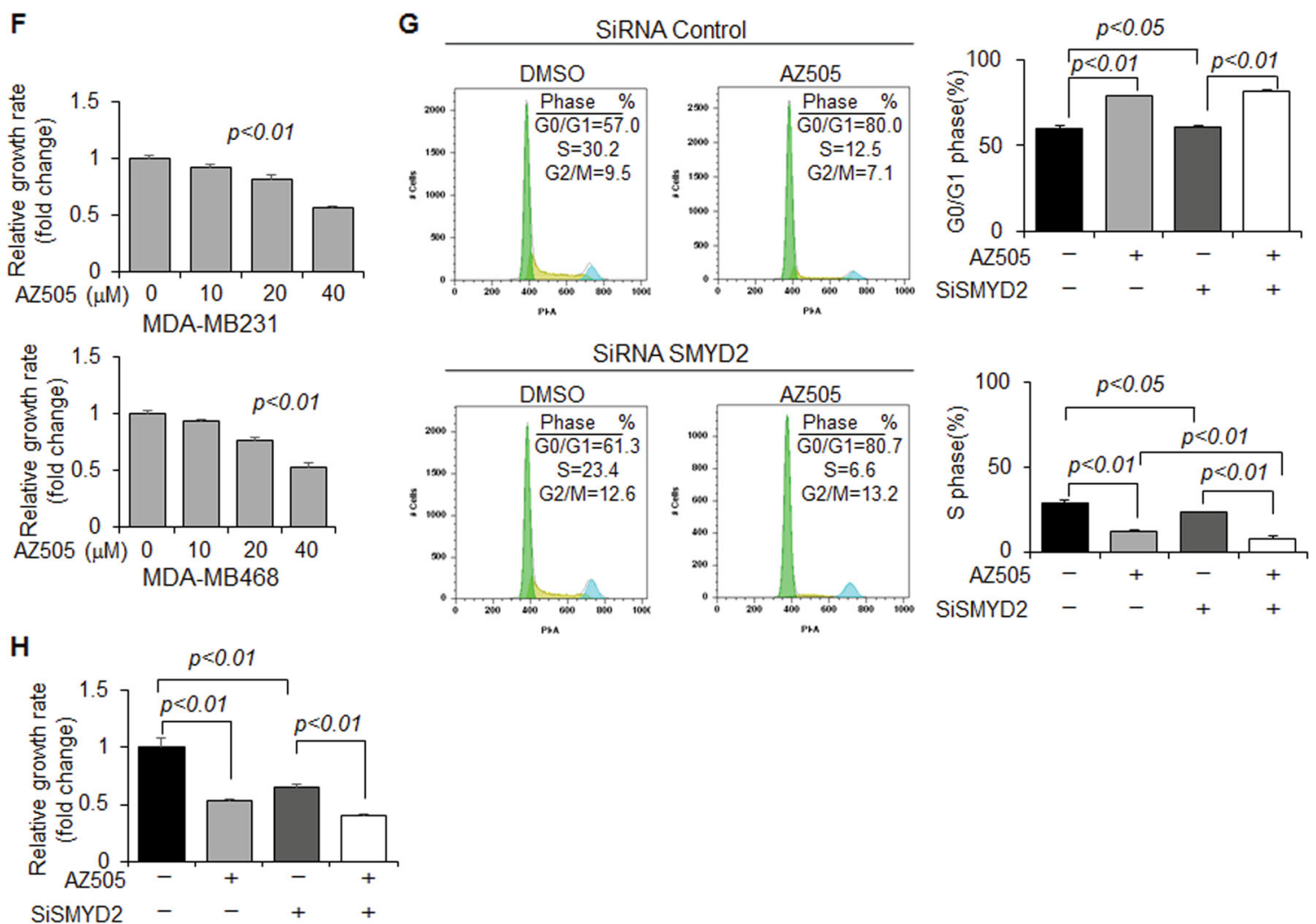

Fig. 3 Inhibition of SMYD2 with its specific inhibitor, AZ505, induced TNBC cell apoptosis but suppressed TNBC cell migration and proliferation in vitro. a AZ505 treatment $(20 \mu \mathrm{M}, 24 \mathrm{~h})$ induced MDA-MB231 cell death, as examined by TUNEL assay. One representative experiment from two independent experiments with similar results was shown. b AZ505 treatment $(20 \mu \mathrm{M}, 24 \mathrm{~h})$ increased Sub-G1 phase in MDAMB468 cells as examined by FACS analysis. $n=3$. c Wound closure assays indicated that AZ505 treatment (24 h) suppressed MDA-MB231 cell migration in a dose dependent manner. $\mathbf{d}$ Would closure assays indicated that knockdown of SMYD2 suppressed MDA-MB231 cell migration, and AZ505 treatment further suppressed these cell migration in SMYD2-knockdown MDA-MB231 cells, $20 \mu \mathrm{M}$ AZ505 was added in the medium $24 \mathrm{~h}$ after siRNA or control was transfected, then cells were incubated for another $24 \mathrm{~h}$. e FACS analysis indicated that inhibition of SMYD2 with AZ505 induced cell-cycle arrest at the G0/G1 phase in MDA-MB231 cells (left panel) and MDA-MB468 cells (right panel). $\mathbf{f}$ Inhibition of SMYD2 with AZ505 inhibited the proliferation of both MDA-MB231 cells and MDA-MB468 cells in a dose dependent manner as examined by MTT assay. The AZ505 was added according to the concentrations shown in the figure for $24 \mathrm{~h}$. $\mathbf{g}$ FACS analysis indicated that knockdown of SMYD2 with siRNA induced cell-cycle arrest at the G0/G1 phase in MDA-MB231 cells, and treatment with AZ505 further induced the cell cycle arrest in SMYD2-knockdown MDA-

MB231 cells. One of three independent experiments was shown. $\mathbf{h}$ Knockdown of SMYD2 inhibited MDA-MB231 cell proliferation as indicated by MTT assay. Treatment with AZ505 only slightly further decreased the proliferation of SMYD2 knockdown MDA-MB231 cells

proliferation of Smyd2 knockout cells (Supplementary Fig. S1,C). These results support the specificity of AZ505 on the inhibition of SMYD2.

We further found that the tumor growth in MDAMB231 implanted female nude mice was significantly retarded by daily intraperitoneal injections of AZ505 at a dose of $40 \mathrm{mg} / \mathrm{kg}$ body weight (Figs. 4a-c). AZ505 treatment also decreased tumor cell proliferation and induced tumor cell apoptosis as seen by Ki67 staining (Fig. 4d) and TUNEL analysis (Fig. 4e). The effect of AZ505 on inhibition of TNBC growth was also confirmed in nude mice with MDA-MB468 xenografts (Figs. 4f-j). Our results suggest that SMYD2 may serve as a potent therapeutic target in TNBC treatment justifying further investigation into the mechanisms of SMYD2 in TNBC development.

\section{SMYD2 interacts with STAT3 and the p65 subunit of NF-KB in TNBC cells}

Triple-negative breast cancers are associated with activation through an unknown mechanism of the JAK2/ STAT3 signaling pathway ${ }^{19}$. Numerous studies have shown that NF- $\mathrm{kB}$ activation in tumors mimics a classical inflammatory response $\mathrm{e}^{20-22}$ and appears to result from upregulation of cytokine gene expression in breast cancer cells. We found that SMYD2 interacts with STAT3 and the p65 subunit of NF- $\mathrm{kB}$ in both MDA-MB231 and MDA-MB468 cells (Figs. 5a, b). 


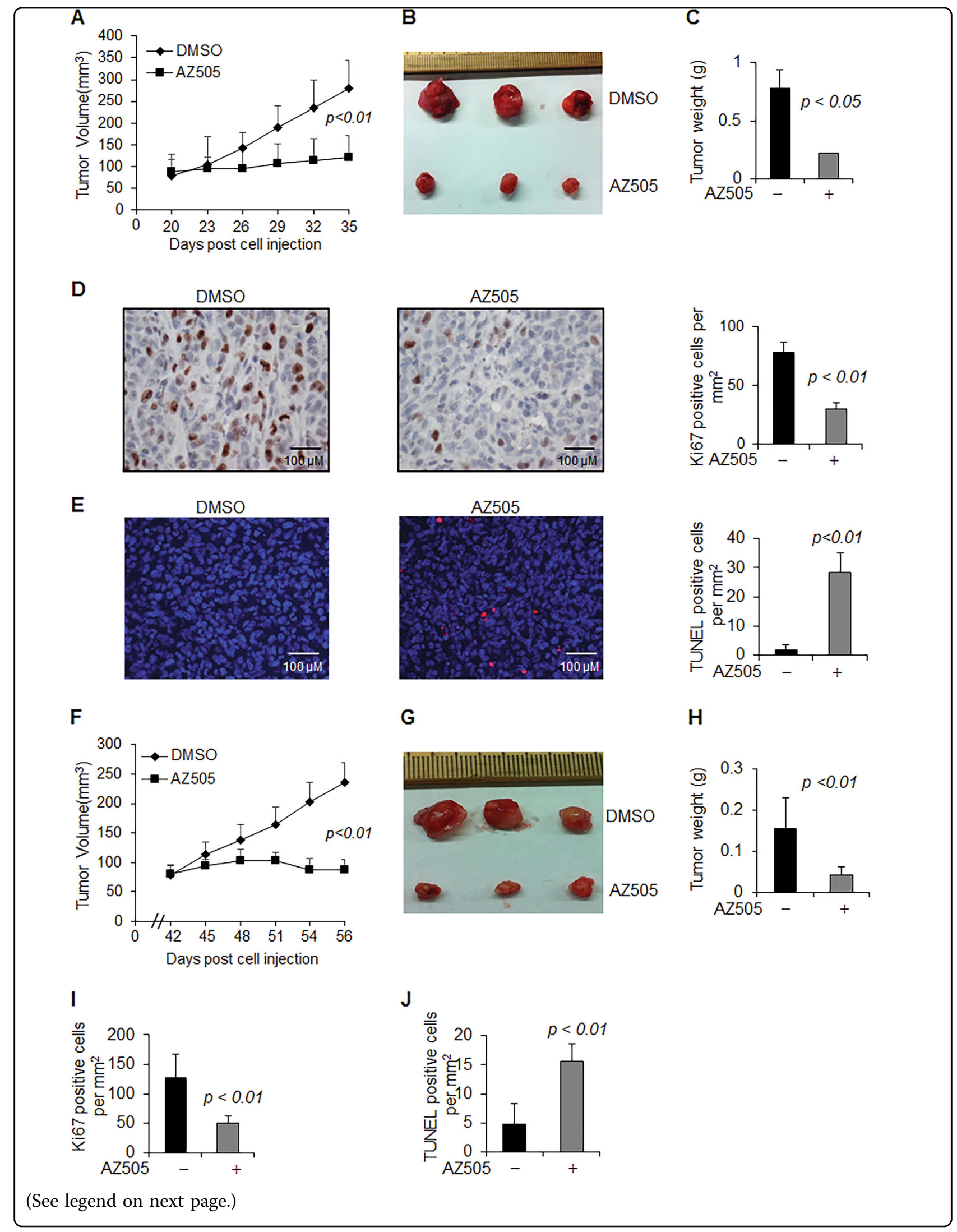




\begin{abstract}
(see figure on previous page)
Fig. 4 Inhibition of SMYD2 with its specific inhibitor, AZ505, delays tumor growth in vivo. a Tumor growth inhibition curve in female athymic nude mice bearing MDA-MB231 tumor xenografts treated intraperitoneally with either vehicle or AZ505 at doses described in Materials and Methods for fourteen consecutive days beginning on day 21 post implantation of $5 \times 10^{6}$ MDA-MB231 human breast cancer cells. Each point represents mean $\pm \mathrm{SD}, n=10$. $\mathbf{b}$ and $\mathbf{c}$ Image (b) and graph (c) depicting the reduction in tumor size and tumor weight observed when MDA-MB231 implanted mice were treated with AZ505 compared to vehicle DMSO treated controls. Unpaired T-test was performed on tumor weight, $p$-value $<0.01, n=10$. $\mathbf{d}$ Tumor cell proliferation was decreased in xenografts from MDA-MB231 implanted mice treated with AZ505 compared to those treated with vehicle DMSO, as examined with Ki67 staining. The percentage of Ki67-positive nuclei was calculated in per $\mathrm{mm}^{2}$. $p$-value $<0.01$. Scale bars, $100 \mu \mathrm{m}$. e AZ505 treatment induced tumor cell death in xenografts from MDA-MB231 implanted mice compared to those mice treated with vehicle DMSO, as examined by TUNEL assay. $p<0.01$. Scale bars, $100 \mu \mathrm{m}$. f Tumor growth inhibition curve in female athymic nude mice bearing MDA-MB468 tumor xenografts treated intraperitoneally with either vehicle or AZ505 at doses described in Materials and Methods for fourteen consecutive days beginning on day 42 post implantation of $5 \times 10^{6}$ MDA-MB468 human breast cancer cells. Each point represents mean $\pm S D, n=5$. $\mathbf{g}$ and $\mathbf{h} I m a g e s$ (g) and graph (h) depicting the reduction in tumor size and tumor weight observed when MDA-MB468 implanted mice are treated with AZ505 compared to vehicle DMSO treated controls. Unpaired T-test was performed on tumor weight, $p<0.01, n=5$. $\mathbf{i}$ Tumor cell proliferation was decreased in xenografts from MDA-MB468 implanted mice treated with AZ505 compared to those treated with vehicle DMSO, as examined with Ki67 staining. The percentage of Ki67-positive nuclei was calculated in per $\mathrm{mm}^{2} . p<0.01$. $\mathbf{j}$ AZ505 treatment induced tumor cell death in xenografts from MDA-MB468 implanted mice treated with AZ505 compared to those mice treated with vehicle DMSO, as detected by TUNEL assay. $p<0.01$
\end{abstract}

\section{SMYD2 regulates the methylation and phosphorylation of STAT3 and the p65 subunit of NF-KB in TNBC cells}

To determine if SMYD2 is involved in methylation of STAT3 and p65, leading to their activation in TNBC cells, we examined whether shRNA knockdown of SMYD2 decreased the levels of methylated STAT3 and p65 in MDA-MB231 and MDA-MB468 cells. Methylation was assessed with an anti-pan methylated lysine antibody. An anti-STAT3 antibody and an anti-p65 antibody were used to pull down STAT3 and p65, which were then blotted with an anti-pan methylated lysine antibody, and the same blot was then stripped and re-probed with the anti-STAT3 and anti-p65 antibodies to confirm their presence. We found that silencing of SMYD2 with shRNA decreased the methylation of STAT3 and p65 in MDA-MB231 (Fig. 5c) and MDAMB468 cells (Supplementary Fig. S3A). Knockdown of SMYD2 with shRNA also decreased the phosphorylation of STAT3 ${ }^{\mathrm{Y} 705}$ and $\mathrm{p} 65^{\mathrm{S} 536}$ while total STAT3 and $\mathrm{p} 65$ expression levels were not changed. However, overexpression of SMYD2 increased the methylation and phosphorylation of STAT3 $^{\mathrm{Y} 705}$ and $\mathrm{p}^{\mathrm{S} 536}$ in HEK293T cells (Supplementary Fig. S3B). We also found that treatment with AZ505 decreased the methylation and phosphorylation of STAT3 and p65 in MDA-MB231 (Fig. 5d) and MDA-MB468 cells (Supplementary Fig. S3C). The phosphorylation of STAT3 and p65 were also decreased in tumors from SMYD2 knockdown (Fig. 5e) and AZ505 treated MDA-MB231 bearing mice (Fig. 5f). Our in vitro methylation assay confirmed that SMYD2 could methylate STAT3 and p65 at K685 and K310, respectively (Supplementary Fig. S4A and S4B). These results suggested that SMYD2 methylation of STAT3 and p65 contributes to the hyper-phosphorylation of STAT3 and p65 in MDA-MB231 and MDA-MB468 cells, which promotes tumor growth ${ }^{23,24}$.
There is a synergistic effect between SMYD2, STAT3, and NF-KB p65 in TNBC cells

STAT3 can modify p65 post-translationally by recruitment of the acetyltransferase p300 to mediate the acetylation of NF- $\mathrm{KB}$ to prolong its nuclear retention ${ }^{25}$. To address the role of STAT3 in SMYD2 mediated NF- $\mathrm{KB}$ activation, we treated TNBC cells with STAT3 shRNA and S3I-201, a commonly used STAT3 inhibitor that binds to the STAT3-SH2 domain to prevent STAT3 phosphorylation, dimerization, and DNA binding ${ }^{26}$. We found that knockdown of STAT3 and inhibition of STAT3 with S3I-201 not only disrupted the interaction between SMYD2 and p65 (Figs. 6a, b, top panel) but also decreased the methylation of p65 as well as the phosphorylation of STAT3 ${ }^{\mathrm{Y} 705}$ and $\mathrm{p} 65^{\mathrm{S} 536}$ in MDA-MB231 cells (Figs. 6a, b). Treatment with the NF- $\mathrm{kB}$ inhibitor, BAY-11-7085, also disrupted the interaction between SMYD2 and STAT3 and decreased the methylation and phosphorylation of STAT3 as well as the phosphorylation of p65 (Fig. 6c). Based on this, we proposed that STAT3 contributes to SMYD2 mediated NF- $\mathrm{KB}$ activation and that p65 also contributes to SMYD2 mediated STAT3 activation.

There are feedback networks among SMYD2, STAT3, NF$K B$, and cytokines in TNBC cells

Both STAT3 and NF-kB p65 are important transcription factors, which regulate gene expression involved in cell proliferation and apoptosis through binding directly to promoters. These two proteins also affect cellular signaling pathways indirectly through their downstream cytokines, in which IL-6 and TNFa have played particularly important roles, because they can form a feedback loop with STAT3 and NF-kB, respectively ${ }^{27,28}$. We found that IL-6 treatment not only increased the phosphorylation of STAT3 but also increased the expression of 


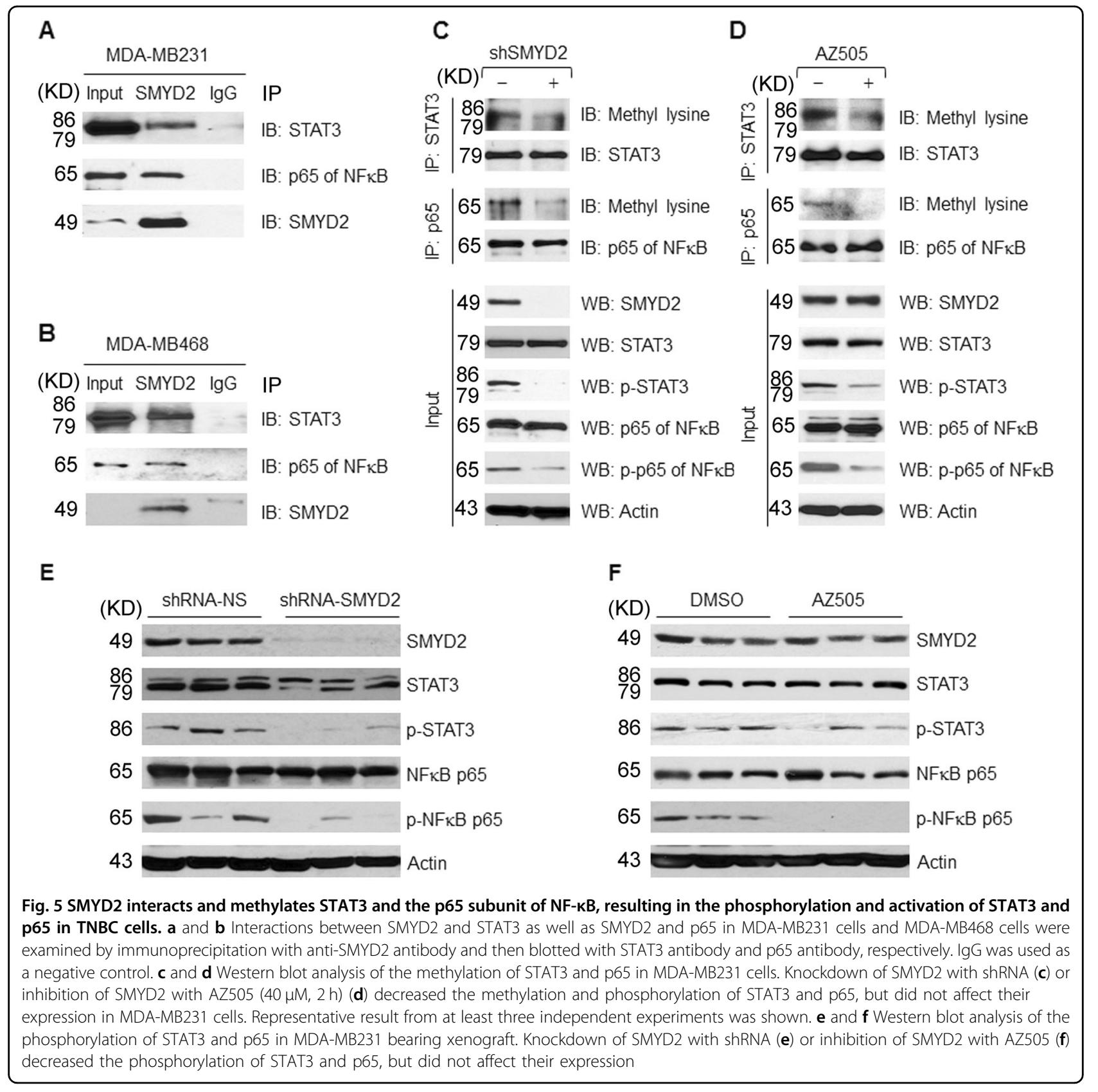

SMYD2 in MCF-10A cells (Fig. 7a), whereas treatment with TNF $\alpha$ also increased the phosphorylation of p65 and the expression of SMYD2 in these cells (Fig. 7b). We also found that treatment with the STAT3 inhibitor, S3I-201 and the NF-kB inhibitor, BAY-11-7085, decreased the expression of SMYD2 in these cells in a dose dependent (Figs. 7c, d) and time dependent (Figs. 7e, f) manner. We further found that STAT3 and p65 bound to the promoter of SMYD2 in MDA-MB231 cells (Fig. 7g). In addition, overexpression of STAT3 and p65 increased the expression of SMYD2 mRNA, and coexpression of STAT3 or p65 with SMYD2 increased the expression of IL-6 and
TNF $\alpha$, respectively (Figs. 7h, i). These results suggest the existing of two positive feedback loops in TNBC cells: SMYD2-IL-6-STAT3-SMYD2 and SMYD2-TNF $\alpha-N F-$ kB-SMYD2.

\section{SMYD2 regulates other signaling pathways in TNBC cells via the methylation of histones}

Our previous ChIP-seq data indicated that PTPN13 was regulated by SMYD2 via the methylation of histones ${ }^{29}$, which regulates a variety of cellular processes and oncogenic transformation by removing phosphate groups from phosphorylated tyrosine residues on a number of proteins, 


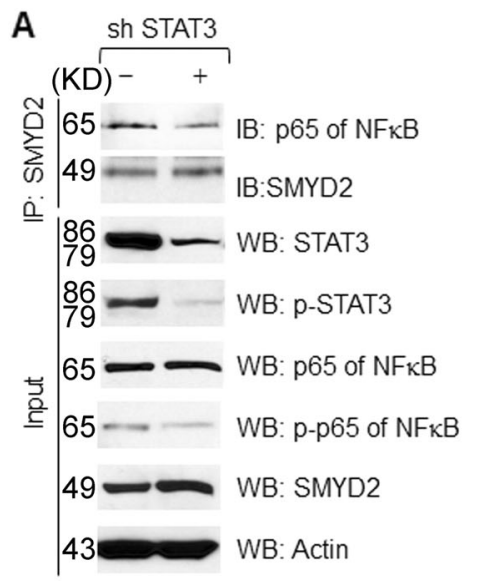

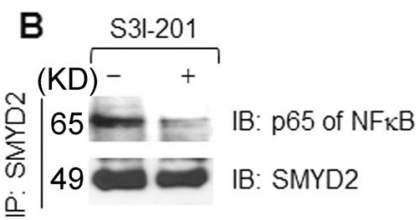
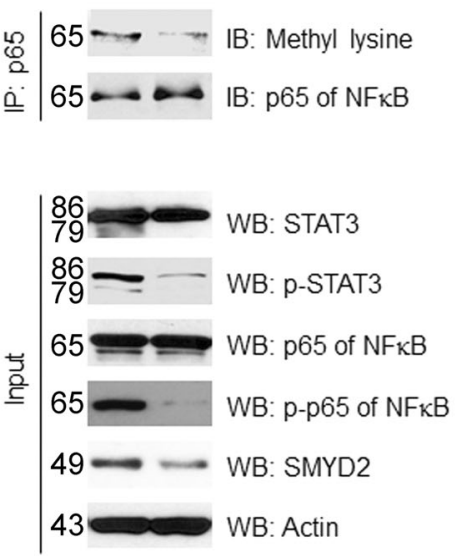
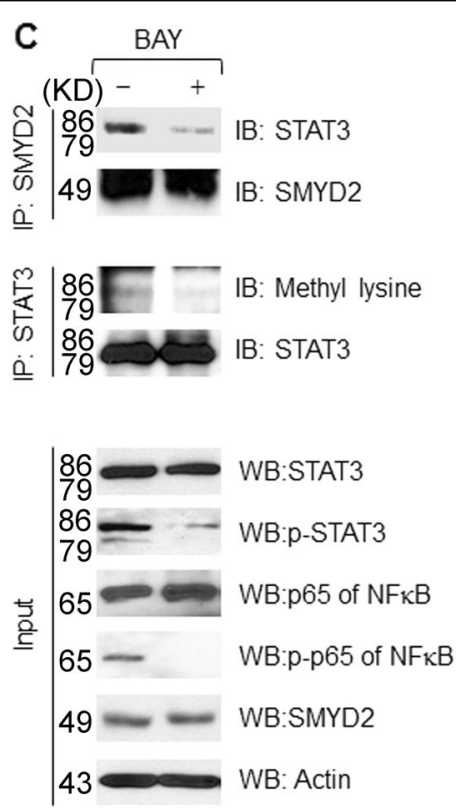

Fig. 6 Synergistic effects exist among SMYD2, STAT3 and NF-KB in MDA-MB231 cells. a Knockdown of STAT3 disrupted the interaction between SMYD2 and p65 and decreased the phosphorylation of p65 in MDA-MB231 cells. b Inhibition of STAT3 with its inhibitor S3I-201(100 $\mu$ M, 3 h) disrupted the interaction between SMYD2 and p65, and decreased the methylation and phosphorylation of p65 as well as the expression of SMYD2 in MDA-MB231 cells. Representative result from at least two independent experiments was shown. c Inhibition of NFKB with its inhibitor BAY-11-7085 $(40 \mu \mathrm{M}, 3 \mathrm{~h})$ disrupted the interaction between SMYD2 and STAT3, and decreased the methylation and phosphorylation of STAT3 in MDA-MB231 cells. Representative result from at least two independent experiments was shown

including ERK and STAT ${ }^{30,31}$. SMYD2 is able to downregulate the expression of PTPN13 in TNBC cells (Supplementary Fig. S5A-S5C), suggesting that SMYD2 mediated downregulation of PTPN13 might be one of the mechanisms responsible for the phosphorylation and activation of these TNBC associated signaling pathways in TNBC cells (Supplementary Fig. S5D and S5E).

\section{Discussion}

The term TNBC came into vogue shortly after the introduction of routine HER-2 testing for breast cancer to describe the clinical subset of breast cancer patients whose tumors tested negative for estrogen receptor, progesterone receptor and HER-2 expression ${ }^{32}$. These patients were notable for their relatively poor prognosis and for their lack of a recognized target for molecularoriented therapy. In the last few years, based on the aberrant signal transduction pathways that may be involved in regulating growth, survival and the development of chemo-resistance in TNBC, a number of alternative strategies have been evaluated in the advanced clinical stages in TNBC patients ${ }^{33-35}$. However, the clinical results have so far been somewhat disappointing. A clear understanding of the molecular pathology of TNBC, including the pathogenesis of the disease and the mechanisms of resistance to currently available therapies, is a matter of urgency and these efforts should provide novel therapeutic targets for TNBC treatment.

In this study, we have demonstrated that SMYD2 is a novel epigenetic regulator associated with TNBC and we have uncovered the underlying mechanisms associated with SMYD2 regulation of TNBC progression (Fig. 8). SMYD2 was found to be significantly expressed at higher levels in TNBC cells both at the mRNA and protein level. We should emphasize that SMYD2 was also upregulated in other types of breast cancer (Fig. 1a). Thus, targeting SMYD2 may also be a potential therapeutic strategy in other types of breast cancers. However, whether the mechanisms identified for SMYD2 in TNBC work in other breast cancer need to be further investigated in the future. In TNBC, we found that upregulation of SMYD2 and the resulting methylation of STAT3 and the p65 subunit of NF- $\mathrm{kB}$ lead to their phosphorylation and activation, impacting cell cycle and apoptosis, respectively. We also found a synergistic cross-talk mechanism involving SMYD2, STAT3, and NF-kB in TNBC cells. STAT3 was shown to contribute to the NF- $\mathrm{B}$ p65 subunit post-translational modification by recruitment of SMYD2, leading to the methylation and activation of p65, while the p65 subunit of NF- $\mathrm{kB}$ could also contribute to the STAT3 posttranslational modification by recruitment of SMYD2, 


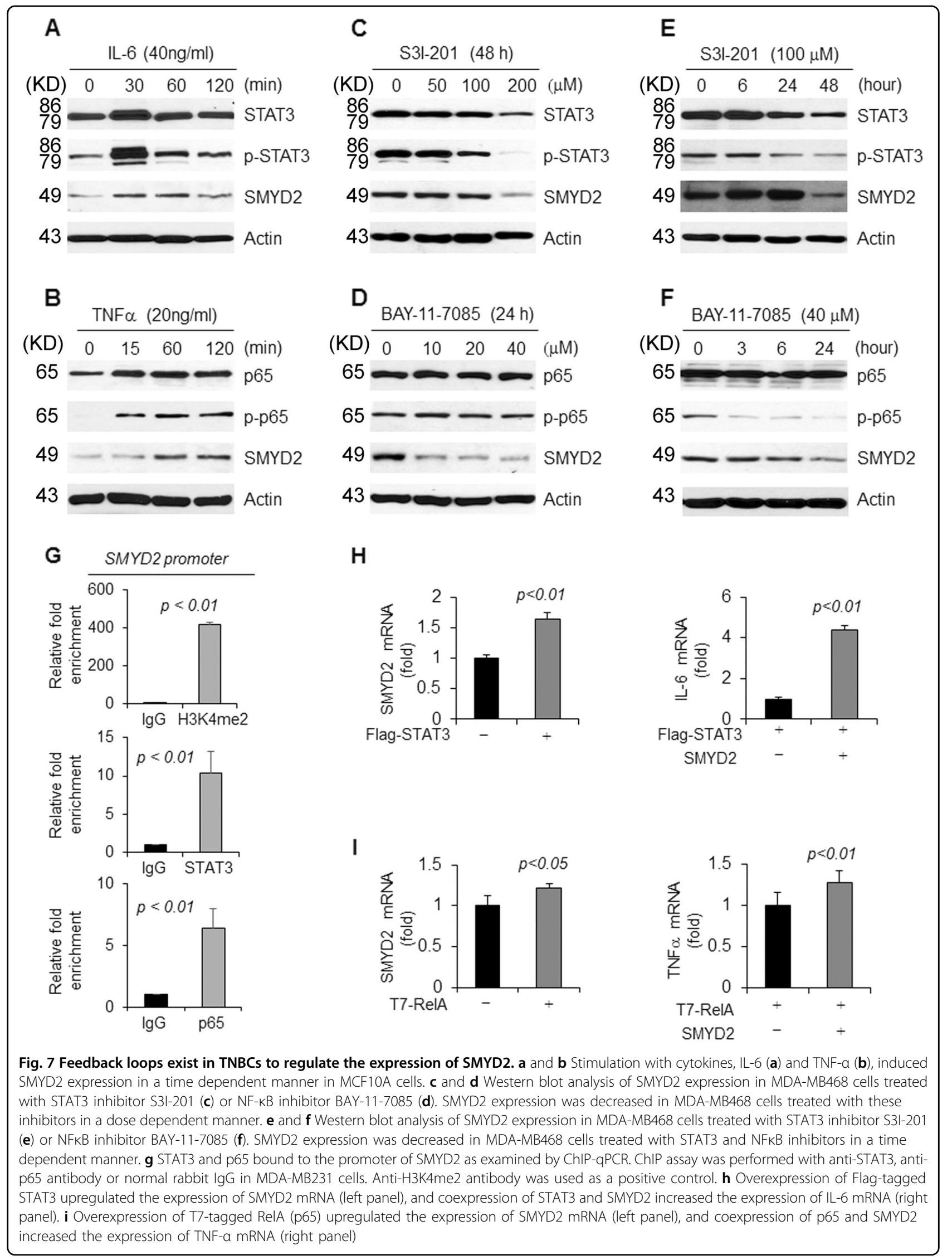




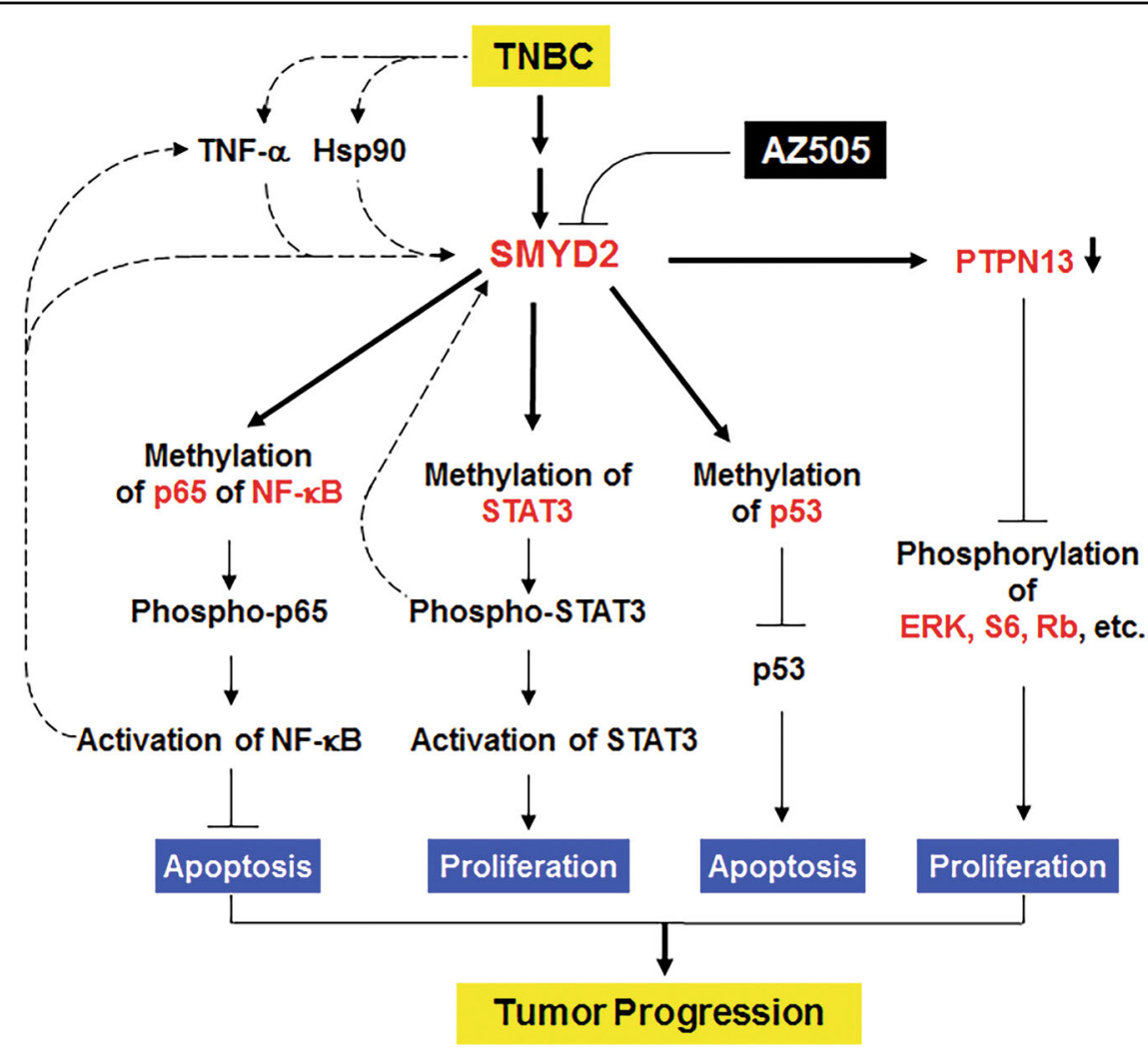

Fig. 8 Working model of SMYD2 in regulation of TNBC progression. A schematic diagram depicting SMYD2 mediated pathways and processes in TNBC. SMYD2 was upregulated in TNBC, which may be stabilized via its interaction with HSP90 and induced by TNF-a secreted by immune cells in the tumor microenviroment or through other unknown mechanisms. Upregulated SMYD2 in TNBC cells, (1) methylates STAT3, leading to its activation and tumor cell proliferation; (2) methylates the p65 subunit of NF-KB, leading to its activation which represses tumor cell apoptosis; (3) methylates p53, leading to the repression of p53 and cystic renal epithelial cell apoptosis; and (4) regulates the transcription of PTPN13, a protein of the protein tyrosine phosphatase (PTP) family, which may regulate the phosphorylation and activation of ERK, mTOR, Akt, and RB signaling. Targeting SMYD2 with its specific inhibitor, AZ505, retarded tumor progression in TNBC cell implanted nude mice. In addition, two positive feedback loops can be observed: SMYD2-IL-6-STAT3-SMYD2, and SMYD2-TNF-a-NF-KB-SMYD2, which may further increase the levels of SMYD2 in TNBC cells

leading to the methylation and activation of STAT3 in TNBC cells. We identified two positive feedback loops, SMYD2-IL-6-STAT3-SMYD2 and SMYD2-TNF $\alpha$-NFKB-SMYD2, which integrated epigenetic regulation with inflammation in TNBC development and suggested that SMYD2 could be further induced by immune cell cytokines, such as IL- 6 and TNF $\alpha$, during TNBC progression. In addition, we confirmed that PTPN13 is a SMYD2 transcriptional target gene in TNBC cells, which may link SMYD2 to other breast cancer associated signaling pathways, including ERK, mTOR, and Akt signaling. The crosstalk and synergistic effects between SMYD2 and its non-histone substrates, STAT3 and NF- $\mathrm{kB}$, could be a general mechanism in human diseases that occurs when SMYD2 is upregulated, suggesting that targeting SMYD2 with its specific inhibitor, AZ505, could be a potential strategy for the treatment of TNBC and other diseases in which there is elevation of SMYD2.
Protein-lysine methyltransferases represent one of several families of enzymes with critical roles in epigenetic regulation, as they catalyze the transfer of methyl groups from $S$-adenosyl-L-methionine to acceptor lysine residues on histone and/or non-histone protein substrates, impacting a variety of biological processes and disease states $^{36,37}$. The SMYD family comprises a subset of five SET domain-containing proteins with unique domain architecture, whereas these proteins have different distributions and functions. SMYD1 was reported as regulator of heart and skeletal muscle development ${ }^{38}$. SMYD3 plays a role in transcriptional regulation and tumorigenesis $^{39}$. SMYD4 was identified as a potential tumor suppressor gene involved in breast cancer development ${ }^{40}$. SMYD5 was identified to be critical in cancer metastasis in breast cancer cells during lung colonization $^{41}$. The expression of SMYD3, which shares a high degree of sequence homology with SMYD2, was significantly lower compared to the significantly higher 
expression of SMYD2 $(p<0.001)$ in basal-like breast tumors ${ }^{4}$. SMYD2 was originally identified as a histone methyltransferase that specifically methylates H3K4 and H3K36 in cooperation with the histone deacetylase complex $^{8}$. Recent studies identified that SMYD2 also methylated key cancer proteins, including RB, p53 and HSP90, ER $\alpha$, and PTEN, to alter their functions during cancer development ${ }^{10,11,13,42,43}$. In this study, we found that STAT3 and the p65 subunit of NF-kB are novel SMYD2 non-histone substrates, and we provided evidence that upregulation of SMYD2 caused methylation of STAT3 and p65, leading to their phosphorylation and activation, which impacted TNBC cell proliferation and apoptosis.

Cytokine-induced JAK-STAT3 signaling has emerged as a positive regulator of cancer ${ }^{44}$. Our results demonstrated that SMYD2 acts as a novel mediator of STAT3 activation via lysine methylation which results in its phosphorylation. Phosphorylation leads to dimerization and nuclear accumulation of STAT3, which then recognizes target DNAs and activates gene expression ${ }^{45}$. In addition, STAT3 activity is also regulated by the association with multiple cofactors.

It has been shown that the p65 subunit of NF- $\mathrm{KB}$ interacts physically with STAT3, facilitating NF-kB recruitment to STAT3 promoters and vice versa, which suggests that NF- $\mathrm{KB}$ and STAT3 may cooperatively regulate a number of target genes ${ }^{46}$. We found that the p65 subunit of NF- $\mathrm{kB}$ is a novel non-histone substrate of SMYD2 in TNBC cells (Fig. 5). Methylation of p65 via SMYD2 also resulted in its phosphorylation and activation, which might regulate TNBC cell proliferation and survival (Fig. 8). We further found that STAT3 can modify p65 post-translationally by recruitment of SMYD2, mediating the methylation of the NF-kB p65 subunit (Fig. 6). This crosstalk among SMYD2, STAT3, and NF-kB in TNBC may be applied to other human diseases which are characterized by upregulation of SMYD2.

$\mathrm{NF}-\mathrm{kB}$, as a transcription factor, can be activated to induce expression of chemokines and cytokines within pre-malignant cells, leading to the recruitment and activation of immune cells. Activated immune cells in turn produce more pro-inflammatory cytokines/chemokines and growth factors, such as interleukin-1 (IL-1), IL-6, and $\mathrm{TNF} \alpha$, in an autocrine and/or paracrine manner to further stimulate NF- $\mathrm{kB}$ activation within the pre-malignant cells, forming a positive feedback loop ${ }^{47}$. We found that IL-6 and TNF $\alpha$ treatment not only induced the phosphorylation and activation of STAT3 and p65, respectively, but also increased the expression of SMYD2 (Figs. 7a, b). In addition, treatment with inhibitors of STAT3 and NF- $\mathrm{kB}$ decreased the expression of SMYD2 in TNBC cells (Figs. 7c-f), and we further found that STAT3 and p65 bound to the promoter of SMYD2 (Fig. $7 \mathrm{~g}$ ) suggesting that two positive feedback loops exist: SMYD2-IL-6STAT3-SMYD2 and SMYD2-TNF $\alpha-N F-\kappa B-S M Y D 2$ in TNBC cells. NF- $\kappa B$ mediated immune cell activation may produce additional pro-inflammatory cytokines/chemokines and growth factors, including IL- 6 , and TNF $\alpha$, in an autocrine and/or paracrine manner during TNBC progression, which may further induce the expression of SMYD2. Thus, our results not only integrate inflammation with epigenetic regulation in TNBC, but also suggest crosstalk between STAT3 and NF-kB in SMYD2 mediated tumor progression. Together, these results suggest that the growth and survival of TNBC cells could be promoted through a synergistic mechanism involving enhanced cytokine signaling and upregulation of SMYD2.

In contrast to the positive feedback between the p65 subunit of NF-kB and STAT3, a mutual inhibition has been reported between $\mathrm{p} 65$ and p53, in that the p65 subunit inhibits p53 dependent transactivation and p53 can suppress NF-kB transcriptional activity ${ }^{48}$. It has also been reported that SMYD2 represses p53 activity through methylation ${ }^{10}$. Our results showing that SMYD2 methylated p65 and activated NF- $\mathrm{BB}$ suggested another mechanism for the mutual inhibition of p65 and p53, which might be mediated by SMYD2.

Novel therapeutic strategies have now reached advanced stages of clinical evaluation in TNBC patients. However, the only targeted agent currently available for patients with TNBC remains the anti-VEGF monoclonal antibody bevacizumab, which has not been specifically designed for TNBC. Our study provides evidence that deregulation of SMYD2 appears to play a crucial role in TNBC. Our study has shown for the first time the effect of the specific SMYD2 inhibitor, AZ505, in an in vivo disease model and it clearly delayed tumor growth in TNBC xenografts implanted nude mice ${ }^{18}$. Given the importance of SMYD2 in TNBC development, SMYD2 should become an attractive candidate for the development of targeted therapy specific for TNBC.

\section{Materials and methods}

\section{Cell culture and reagents}

Human breast cancer cell lines MCF-7, MDA-MB231, MDA-MB468, Hs578T and non-tumorigenic MCF10A human mammary epithelial cells as well as Human Embryonic Kidney 293 cells (HEK293) were obtained from American Type Culture Collection. T47D cells was kindly provided by Dr. Joan Lewis-Wambi and Dr. Christy Hagan at the University of Kansas Medical Center (KUMC) (Kansas City, Kansas, USA). All of the cells were maintained according to the recommended protocol. For knockdown of SMYD2, cell lines were infected with lentiviral pGIPZ shRNA vector containing short hairpins and GFP reporter (Open Biosystems, Pittsburgh, PA, USA). AZ505 was purchased from MedChem Express and 
dissolved in DMSO (Sigma) at a stock solution of $20 \mathrm{mM}$. S3I-201 was purchased from Sigma and dissolved in DMSO (Sigma) at a stock solution of $100 \mathrm{mM}$. BAY-117085 was purchased from Cayman Chemical and dissolved in DMSO (Sigma) at a stock solution of $40 \mathrm{mM}$. All the stock solutions were stored at $-20^{\circ} \mathrm{C}$. TNF- $\alpha$ and IL6 were purchased from Sigma-Aldrich. pAcGFP1-C1SMYD2 and pAcGFP1-C1 plasmids were purchased from Clonetech.

\section{Western blot and immunoprecipitation}

We performed immunoprecipitation and Western blotting on whole-cell lysates as previous described ${ }^{49}$. Briefly, cell pellets were collected and re-suspended in lysis buffer (20 mM Tris- $\mathrm{HCl}, \mathrm{pH} 7.4,150 \mathrm{mM} \mathrm{NaCl}, 10 \%$ glycerol, $1 \%$ Triton X-100, $1 \mathrm{mM} \mathrm{Na} \mathrm{VO}_{4}, 25 \mathrm{mM} \beta$-glycerol-phosphate, $0.1 \mathrm{mM}$ PMSF, Roche complete protease inhibitor set and Sigma phosphatase inhibitor set). The resuspended cell pellet was vortexed for $20 \mathrm{~s}$ and then incubated on ice for $30 \mathrm{~min}$ and centrifuged at 20,000 $\mathrm{g}$ for $30 \mathrm{~min}$. The protein in the cell lysates were subjected to Western blot analysis or immunoprecipitation.

For SMYD2 immunoprecipitation, anti-SMYD2 antibody or control IgG was coupled to protein A agarose bead (Pierce) in PBS containing $5 \mathrm{mg} / \mathrm{ml}$ bovine serum albumin (Sigma) for $6 \mathrm{~h}$ at $4{ }^{\circ} \mathrm{C}$ on a rotating platform. The cell lysates were then incubated with the beads coupled with SMYD2 antibody or control IgG overnight at $4{ }^{\circ} \mathrm{C}$. The next day, beads were washed with lysis buffer containing additional $300 \mathrm{mM} \mathrm{NaCl}$ and the immunoprecipitants were eluted off the beads using loading buffer with boiling for $5 \mathrm{~min}$.

The antibodies used for Western analysis included: antiSMYD2, anti-STAT3, anti-p65 antibodies (Santa Cruz,1:500 dilution), anti-actin antibody (Sigma, 1:5000 dilution), anti-phospho-STAT3, anti-phospho-p65, antiphospho-S6, anti-phospho-AKT, anti-phospho-ERK, antiS6, anti-AKT, and anti-ERK antibodies (Cell Signaling Technologies; 1:1000 dilution). All primary antibodies were used at 1:50 dilution for immunoprecipitation and for Western blotting as indicated above. Donkey-antirabbit IgG-horseradish peroxidase, Donkey-anti-goat IgG-horseradish peroxidase and Goat anti-mouse IgGhorseradish peroxidase (Santa Cruz, 1:8000 dilution) were used as secondary antibodies.

\section{Immunohistochemistry}

Xenograft tissues were fixed with $4 \%$ paraformaldehyde (pH 7.4). For Ki67 staining, a monoclonal mouse anti-Ki67 antibody (Cell Signaling Technologies; 1:1000 dilution), a biotinylated secondary antibody (Sigma-Aldrich; 1:100 dilution), and DAB substrate system were used. Then sections were counter stained by hematoxylin. Images were analyzed with a NIKON ECLIPSE 80i microscope.

\section{Immunofluorescence staining}

Breast cancer cell lines as well as MCF10A cells were detected by immunofluorescence staining (IF staining) with anti-SMYD2 antibody. Cultured cells were grown on sterile glass cover slips overnight at $37^{\circ} \mathrm{C}$. The cells were washed briefly with PBS and then fixed for $10 \mathrm{~min}$ in -20 ${ }^{\circ} \mathrm{C}$ methanol, $2 \mathrm{~min}$ in cold acetone. After that the cells were washed with PBS for three times. Use suction to remove reagents after each step, but avoid drying of specimens between steps. Incubate with primary antibody for $60 \mathrm{~min}$. The antibody is used at $1 \mu \mathrm{g} / \mathrm{ml}$ with $2 \%$ BSA in PBS. Wash three times with PBS for $5 \mathrm{~min}$ each. Incubate with secondary antibody, Fluro-488 anti-goat IgG, for 60 min Wash three times with PBS for 5 min each and then the cover slips were mounted in Prolong Gold Anti-fade reagent with DAPI (Invitrogen). Images were analyzed using a NIKON ECLIPSE 80i microscope.

\section{SMYD2 knockdown by lentivirus carrying SMYD2 ShRNA}

HEK293T cells were transfected either with lentiviral plasmid pGIPZ-SMYD2 (Open Biosystems), carrying SMYD2 shRNA, or with control empty vector pGIPZ-NS, plus psPAX2 packaging plasmid and pMD2.G envelope plasmid using calcium phosphate. After transfection for $16 \mathrm{~h}$, the medium containing the transfection reagent was removed and replaced with fresh complete DMEM plus $10 \%$ FBS and penicillin/streptomycin. The lentiviral particles were harvested from HEK293T cells after another $48 \mathrm{~h}$. MDA-MB231 and MDA-MB468 cells were then infected with appropriate amounts of lentiviral particles together with $5 \mu \mathrm{g} / \mathrm{ml}$ polybrene (Sigma-Aldrich) for $24 \mathrm{~h}$, and then virus-containing medium was removed and replaced with fresh medium plus $10 \mu \mathrm{g} / \mathrm{ml}$ puromycin. After $48 \mathrm{~h}$ of puromycin selection, all remaining cells were GFP positive, as detected by microscopy. MDA-MB231 and MDA-MB468 cells were harvested after lentiviral particle infection for 5 days and analyzed by RT-PCR to examine the efficiency of SMYD2 knockdown. STAT3 knockdown was performed in the same way.

\section{RNA interference}

The RNA oligonucleotides that specifically targeted human SMYD2 was purchased from Santa Cruz Biotechnology, Inc. The RNA oligonucleotides were transfected with DharmaFECT siRNA transfection reagent (Dharmacon). 24 and $48 \mathrm{~h}$ after transfection, cells were harvested and analyzed by Western blotting.

\section{Quantitative reverse-transcription polymerase chain reaction (qRT-PCR)}

Total RNA was extracted using the RNeasy plus mini kit (Qiagen). Total RNA $(1 \mu \mathrm{g})$ was used for RT reactions in a $20-\mu$ reaction to synthesize cDNA using Iscript cDNA Synthesis Kit (BioRad). RNA expression profiles were 
analyzed by real-time PCR using iTaq SYBER Green Supermix with ROX (BioRad) in an icycleriQTM Realtime PCR detection system. The complete reactions were subjected to the following program of thermal cycling: 40 cycles of $10 \mathrm{~s}$ at $95^{\circ} \mathrm{C}$ and $20 \mathrm{~s}$ at $60^{\circ} \mathrm{C}$. A melting curve was run after the PCR cycles, followed by a cooling step. Each sample was run in triplicate in each experiment, and each experiment was repeated 3 times. Expression levels target genes were normalized to the expression level of GAPDH. All the primers used were listed in Table S1.

\section{Chromatin immunoprecipitation (CHIP) assay}

ChIP assay was performed according to the protocol ${ }^{50}$. Chromatin DNA was subjected to IP with anti-Smyd2, anti-STAT3, anti-p65 antibodies and anti-H3K4-me2 antibody (ab7766; Abcam), or normal rabbit IgG and then washed, after which the DNA-protein cross-links were reversed. The recovered DNA was analyzed by PCR for the binding of STAT3, p65, and H3K4-me at the mouse Smyd2 promoter.

\section{Terminal deoxynucleotidyl transferase-mediated dUTP nick end-labeling (TUNEL) assay}

TUNEL assays for AZ505-treated or SMYD2 knockdown cells and for AZ505-treated or shRNA-SMYD2 knockdown xenografts were performed according to the manufacturer's protocols (In Situ Death Detection Kit; Roche). Prolong Gold Anti-fade reagent with DAPI (Invitrogen) was used. Immunofluorescence images were obtained with a NIKON ECLIPSE 80i microscope.

\section{Cell cycle analysis}

A total of 150,000 cells were seeded in six-well plates and allowed to recover for 16-24 $\mathrm{h}$, cells were then starved with serum-free medium for $24 \mathrm{~h}$. Then cells were left untreated or treated with AZ505 for $24 \mathrm{~h}$. The cells were pelleted and washed by adding $1 \mathrm{ml}$ PBS; the cells were centrifuged at $2000 \mathrm{rpm}$ for $5 \mathrm{~min}$ and the supernatant was aspirated; $1 \mathrm{ml}$ of cold $70 \%$ ethanol was added drop by drop into the cell pellet to re-suspend the cells. The cells were then incubated at $-20^{\circ} \mathrm{C}$ overnight. After that the cells were washed twice in $1 \times \mathrm{PBS}$ to remove the ethanol, then centrifuged for $5 \mathrm{~min}$ at $3000 \mathrm{rpm}$ and aspirate the supernatant. For PI staining, the cells were suspended in $0.5 \mathrm{ml}$ of PI buffer (containing RNase and Triton X-100) for 10-15 min at room temperature. The cells were kept away from light at $4{ }^{\circ} \mathrm{C}$ prior to further analysis on the flow cytometer which took place within $1 \mathrm{~h}$. FACS data were analyzed with FlowJo software.

\section{Transfection assays}

HEK293 T cells were cultured to confluence in DMEM media containing $10 \%$ fetal calf serum (FetalcloneIII, Clonetech). The control or SMYD2 overexpression
pAcGFPC1 vectors were transfected into cells using the Lipofectamine 2000 Kit according to the manufacturer's recommendations. Transfected cells were maintained at $37^{\circ} \mathrm{C} / 5 \% \mathrm{CO} 2$ for 24 or $48 \mathrm{~h}$.

\section{MTT assays}

Cell proliferation was measured using MTT-based kit (Promega), according to the manufacturer's instructions.

\section{Wound closure assay}

MDA-MB231 cells or MDA-MB468 cells $\left(4 \times 10^{5}\right.$ cells/ $2 \mathrm{ml}$ ) were seeded in a 6-well plate and incubated at $37^{\circ} \mathrm{C}$ until 90 to $100 \%$ confluent. Wounds were generated on the monolayer of cells with sterile $10 \mu$ pipet tip and washed with PBS to take images at $\mathrm{T}_{0}$, then cells were switched to the serum containing normal medium with different concentrations of AZ505 for $24 \mathrm{~h}$. Pictures of same position of each well were taken at $24 \mathrm{~h}\left(\mathrm{~T}_{24}\right)$. We performed the wound closure assay with MCF-7 cells and T47D cells in a similar way, but pictures were taken at zero $\left(\mathrm{T}_{0}\right)$ and $48 \mathrm{~h}\left(\mathrm{~T}_{48}\right)$, due to the cell growth rates.

\section{In vitro methylation assay}

We made constructs for GST-SMYD2, GST-STAT3 and GST-p65 as well as the lysine site mutants of STAT3 and p65, induced expression in the presence of IPTG, and purified the proteins by glutathione agarose beads. In vitro methylation assays were performed using 1-2 $\mu \mathrm{g}$ of recombinant proteins incubated with $1 \mu \mathrm{g}$ of recombinant GST-SMYD2 and $2 \mu \mathrm{Ci}{ }^{3} \mathrm{H}$-AdoMet (Perkin Elmer) in buffer containing $50 \mathrm{mM}$ Tris- $\mathrm{HCl}(\mathrm{pH} 8.0), 10 \%$ glycerol, $20 \mathrm{mM} \mathrm{KCl}, 5 \mathrm{mM} \mathrm{MgCl} 2$, and $1 \mathrm{mM} \mathrm{PMSF}$ at room temperature overnight. The reaction mixture was resolved by SDS-PAGE followed by autoradiography.

\section{Tumorigenesis and treatment assay}

All animal works were done in accordance with approved protocol by the Institutional Animal Care and Use Committee (IACUC) of the University of Kansas Medical Center. Animals were maintained and treated under pathogen-free conditions. Female NU/NU nude (Charles River) of 4-6 weeks old were used in xenograft studies orthotopic mammary fat pad tumor assays. One shRNA construct showed most effective knockdown of SMYD2 and subsequently used to knockdown SMYD2 in TNBC MDA-MB231 cells as well as the control shRNA cells that were used for animal studies. For orthotopic tumor assays, cells were collected in PBS and inoculated into the right mammary fat pad $\left(5 \times 10^{7} / \mathrm{ml}, 0.1 \mathrm{ml}\right.$ per mouse, $n=6$ in each group). The primary tumor outgrowth was monitored every two or three days with caliper measurements. Tumor volumes were calculated according to the following equation: Volume $=(\text { width })^{2} \times$ length/2. The animals in both groups were euthanized 
when the tumors reached an average length of $1 \mathrm{~cm}$ in control group, and tumors were removed and weighed.

For treatment assay, all the mice were inoculated with MDA-MB231cells $\left(5 \times 10^{7} / \mathrm{ml}, 0.1 \mathrm{ml}\right.$ per mouse, $n=10$ in each group) or MDA-MB468 cells $\left(5 \times 10^{7} / \mathrm{ml}, 0.1 \mathrm{ml}\right.$ per mouse, $n=5$ in each group) via mammary fat pad, tumor growth was monitored. When tumors reached an average volume of about $100 \mathrm{~mm}^{3}\left(50 \sim 200 \mathrm{~mm}^{3}\right)$ in size, then mice were randomized into two groups, DMSO control and SMYD2 inhibitor AZ505 treatment group. AZ505 and DMSO control were administered intraperitoneally at 40 $\mathrm{mg} / \mathrm{kg} / \mathrm{d}$. Tumor size and body weight were recorded every 2 days. Animals were euthanized 2 weeks after the first treatment and tumors were removed and weighed.

\section{TCGA data analysis}

All TCGA data and figures were accessed, analyzed and generated using the cBio Cancer Genomics Portal. All data included in this manuscript is in agreement with the TCGA publication guidelines.

\section{Statistics}

All data are presented as mean \pm SEM. All statistical analyses were performed using SPSS Statistics 22 software. Pvalues were calculated by two-tailed unpaired Student's $t$-test, one-way ANOVA and $p<0.05$ was considered significant.

\section{Study approval}

All animal protocols were approved and conducted in accordance with Laboratory Animal Resources of KUMC (Kansas City, Kansas, USA) and Institutional Animal Care and Use Committee regulations (Protocol \# 2015-2290).

\section{Acknowledgements}

We acknowledge Dr. Joan Lewis-Wambi and Dr. Christy Hagan for providing us the T47D cells. We acknowledge Dr. Bruno Hagenbuch for helping us with the in vitro methylation assays. X. Li acknowledges support from National Institutes of Health grant R01 DK084097 and P30 DK106912. J.P. Calvet acknowledges support from the PKD Foundation and from the Kansas Research and Translation Core Center (P30 DK106912). A.K. Godwin and R. Jensen acknowledge support from National Institutes of Health grant P30 CA168524. L.X. Li acknowledges support from a post-doctoral fellowship funded by an Institutional Development Award (IDeA) from the National Institute of General Medical Sciences (P20 GM103418). J.X. Zhou acknowledges support from the NIH K01 DK107729.

\section{Author details}

'Department of Internal Medicine, University of Kansas Medical Center, Kansas City, KS 66160, USA. ²Department of Biochemistry and Molecular Biology, University of Kansas Medical Center, Kansas City, KS 66160, USA. ${ }^{3}$ Department of Cancer Biology, University of Kansas Medical Center, Kansas City, KS 66160, USA. ${ }^{4}$ Department of Pathology \& Laboratory Medicine, University of Kansas Medical Center, Kansas City, KS 66160, USA

\section{Conflict of interest}

The authors declare that they have no conflict of interest.

\section{Publisher's note}

Springer Nature remains neutral with regard to jurisdictional claims in published maps and institutional affiliations.
Supplementary Information accompanies this paper at https://doi.org/ 10.1038/s41419-018-0347-x.

Received: 21 August 2017 Revised: 6 January 2018 Accepted: 22 January 2018

Published online: 27 February 2018

\section{References}

1. Carey, L., Winer, E., Viale, G., Cameron, D. \& Gianni, L. Triple-negative breast cancer: disease entity or title of convenience? Nat. Rev. Clin. Oncol. 7, 683-692 (2010).

2. Karsli-Ceppioglu, S. et al. Epigenetic mechanisms of breast cancer: an update of the current knowledge. Epigenomics 6, 651-664 (2014).

3. Claude-Taupin, A., Boyer-Guittaut, M., Delage-Mourroux, R. \& Hervouet, E. Use of epigenetic modulators as a powerful adjuvant for breast cancer therapies. Methods Mol. Biol. 1238, 487-509 (2015).

4. Liu, L., Kimball, S., Liu, H., Holowatyj, A. \& Yang, Z. Q. Genetic alterations of histone lysine methyltransferases and their significance in breast cancer. Oncotarget 6, 2466-2482 (2015).

5. Roy, D. M., Walsh, L. A. \& Chan, T. A. Driver mutations of cancer epigenomes. Protein \& Cell 5, 265-296 (2014).

6. Shen, H. \& Laird, P. W. Interplay between the cancer genome and epigenome. Cell 153, 38-55 (2013)

7. Tian, X. et al. Histone lysine-specific methyltransferases and demethylases in carcinogenesis: new targets for cancer therapy and prevention. Curr. Cancer Drug Targets 13, 558-579 (2013).

8. Brown, M. A., Sims, R. J. 3rd, Gottlieb, P. D. \& Tucker, P. W. Identification and characterization of Smyd2: a split SET/MYND domain-containing histone H3 lysine 36-specific methyltransferase that interacts with the $\operatorname{Sin} 3$ histone deacetylase complex. Mol. Cancer 5, 26 (2006).

9. Abu-Farha, M. et al. The tale of two domains: proteomics and genomics analysis of SMYD2, a new histone methyltransferase. Mol. Cell. Proteom. 7, 560-572 (2008).

10. Huang, J. et al. Repression of p53 activity by Smyd2-mediated methylation. Nature 444, 629-632 (2006).

11. Saddic, L. A. et al. Methylation of the retinoblastoma tumor suppressor by SMYD2. J. Biol. Chem. 285, 37733-37740 (2010).

12. Voelkel, T. et al. Lysine methyltransferase Smyd2 regulates Hsp90-mediated protection of the sarcomeric titin springs and cardiac function. Biochim. Et. Biophys. Acta 1833, 812-822 (2013).

13. Zhang, $X$. et al. Regulation of estrogen receptor alpha by histone methyltransferase SMYD2-mediated protein methylation. Proc. Natl. Acad. Sci. USA 110, 17284-17289 (2013).

14. Komatsu, S. et al. Overexpression of SMYD2 relates to tumor cell proliferation and malignant outcome of esophageal squamous cell carcinoma. Carcinogenesis 30, 1139-1146 (2009).

15. Skawran, B. et al. Gene expression profiling in hepatocellular carcinoma: upregulation of genes in amplified chromosome regions. Mod. Pathol. 21, 505-516 (2008).

16. Gao, J. et al. Integrative analysis of complex cancer genomics and clinical profiles using the cBioPortal. Sci. Signal. 6, pl1 (2013).

17. Cerami, E. et al. The cBio cancer genomics portal: an open platform for exploring multidimensional cancer genomics data. Cancer Discov. 2, 401-404 (2012).

18. Ferguson, A. D. et al. Structural basis of substrate methylation and inhibition of SMYD2. Structure 19, 1262-1273 (2011).

19. Marotta, L. L. et al. The JAK2/STAT3 signaling pathway is required for growth of CD44(+)CD24(-) stem cell-like breast cancer cells in human tumors. J. Clin. Investig. 121, 2723-2735 (2011).

20. He, G. \& Karin, M. N. F.-kappaB. and STAT3 - key players in liver inflammation and cancer. Cell Res. 21, 159-168 (2011).

21. Haffner, M. C., Berlato, C. \& Doppler, W. Exploiting our knowledge of NFkappaB signaling for the treatment of mammary cancer. J. Mammary Gland Biol. neoplasia 11, 63-73 (2006).

22. Singh, S. et al. Nuclear factor-kappaB activation: a molecular therapeutic target for estrogen receptor-negative and epidermal growth factor receptor family receptor-positive human breast cancer. Mol. Cancer Ther. 6, 1973-1982 (2007).

23. Yu, H., Pardoll, D. \& Jove, R. STATs in cancer inflammation and immunity: a leading role for STAT3. Nat. Rev. Cancer 9, 798-809 (2009). 
24. Karin, M. \& Greten, F. R. NF-kappaB: linking inflammation and immunity to cancer development and progression. Nat. Rev. Immunol. 5, 749-759 (2005).

25. Lee, $\mathbf{H}$. et al. Persistently activated Stat3 maintains constitutive NF-kappaB activity in tumors. Cancer Cell 15, 283-293 (2009).

26. Siddiquee, K. et al. Selective chemical probe inhibitor of Stat3, identified through structure-based virtual screening, induces antitumor activity. Proc. Natl. Acad. Sci. USA 104, 7391-7396 (2007).

27. Wang, Y., van Boxel-Dezaire, A. H., Cheon, H., Yang, J. \& Stark, G. R. STAT3 activation in response to $\mathrm{IL}-6$ is prolonged by the binding of $\mathrm{IL}-6$ receptor to EGF receptor. Proc. Natl. Acad. Sci. USA 110, 16975-16980 (2013).

28. Oeckinghaus, A. \& Ghosh, S. The NF-kappaB family of transcription factors and its regulation. Cold Spring Harb. Perspect. Biol. 1, a000034 (2009).

29. Li, L. X. et al. Lysine methyltransferase SMYD2 promotes cyst growth in autosomal dominant polycystic kidney disease. J. Clin. Invest 127, 2751-2764 (2017).

30. Alonso, A. et al. Protein tyrosine phosphatases in the human genome. Cell 117, 699-711 (2004)

31. Sardina, J. L. et al. PTPN13 regulates cellular signalling and beta-catenin function during megakaryocytic differentiation. Biochim. Et. Biophys. Acta 1843 2886-2899 (2014)

32. Brenton, J. D., Carey, L. A., Ahmed, A. A. \& Caldas, C. Molecular classification and molecular forecasting of breast cancer: ready for clinical application? J. Clin. Oncol. 23, 7350-7360 (2005).

33. Crown, J., O'Shaughnessy, J. \& Gullo, G. Emerging targeted therapies in triplenegative breast cancer. Ann. Oncol. 23, vi56-vi65 (2012). Suppl 6.

34. Gluz, O. et al. Triple-negative breast cancer--current status and future directions. Ann. Oncol. 20, 1913-1927 (2009).

35. Perou, C. M. et al. Molecular portraits of human breast tumours. Nature $\mathbf{4 0 6}$ 747-752 (2000)

36. Greer, E. L. \& Shi Y. Histone methylation: a dynamic mark in health, disease and inheritance. Nat. Rev. Genet. 13, 343-357 (2012).

37. Joshi, C. P. \& Chiang, V. L. Conserved sequence motifs in plant S-adenosyl-Lmethionine-dependent methyltransferases. Plant Mol. Biol. 37, 663-674 (1998).
38. Gottlieb, P. D. et al. Bop encodes a muscle-restricted protein containing MYND and SET domains and is essential for cardiac differentiation and morphogenesis. Nat. Genet 31, 25-32 (2002).

39. Hamamoto, $R$ et al. SMYD3 encodes a histone methyltransferase involved in the proliferation of cancer cells. Nat. Cell Biol. 6, 731-740 (2004).

40. $\mathrm{Hu}, \mathrm{L}$., Zhu, Y. T., Qi, C. \& Zhu, Y. J. Identification of Smyd4 as a potential tumor suppressor gene involved in breast cancer development. Cancer Res 69, 4067-4072 (2009)

41. Gao, H. et al. Forward genetic screens in mice uncover mediators and suppressors of metastatic reactivation. Proc. Natl. Acad. Sci. USA 111, 16532-16537 (2014).

42. Hamamoto, R., Toyokawa, G., Nakakido, M., Ueda, K. \& Nakamura, Y. SMYD2-dependent HSP90 methylation promotes cancer cell proliferation by regulating the chaperone complex formation. Cancer Lett. 351, 126-133 (2014)

43. Piao, L. et al. The histone methyltransferase SMYD2 methylates PARP1 and promotes poly(ADP-ribosyl)ation activity in cancer cells. Neoplasia 16, 257-264 (2014). 264 e252.

44. Qin, S., Zou, Y. \& Zhang, C. L. Cross-talk between KLF4 and STAT3 regulates axon regeneration. Nat. Commun. 4, 2633 (2013).

45. Shuai, K. \& Liu, B. Regulation of JAK-STAT signalling in the immune system. Nat. Rev. Immunol. 3, 900-911 (2003).

46. Xiao, G. \& Fu, J. NF-kappaB and cancer: a paradigm of Yin-Yang. Am. J. Cancer Res. 1, 192-221 (2011).

47. Karin, M. NF-kappaB as a critical link between inflammation and cancer. Cold Spring Harb. Perspect. Biol. 1, a000141 (2009).

48. Xiao, G. \& Sun, S. C. Activation of IKKalpha and IKKbeta through their fusion with HTLV-I tax protein. Oncogene 19, 5198-5203 (2000).

49. Li, X. et al. Polycystin-1 and polycystin-2 regulate the cell cycle through the helix-loop-helix inhibitor Id2. Nat. Cell Biol. 7, 1202-1212 (2005).

50. Schmidt, D. et al. ChIP-seq: using high-throughput sequencing to discover protein-DNA interactions. Methods 48, 240-248 (2009). 